\title{
66-jährige Patientin mit Glaskörperblutung und Schleimhautblutungen
}

\author{
Stefanie Ackerstaff, Rita Dittmer, Christoph Sucker
}

Neu aufgetretene Schleimhautblutungen veranlassen eine Patientin, sich im Gerinnungszentrum vorzustellen. Acht Monate später hat sie weiterhin Nasenbluten. Außerdem ist eine Glaskörperblutung aufgetreten - ein Symptom, das bei der zu Grunde liegenden Erkrankung sehr selten auftritt.

\section{Erste Vorstellung}

Einer 66-jährigen Patientin sind vor drei Monaten nach einer Zahnsteinentfernung erstmals verlängerte Nachblutungen aufgefallen. Ferner kam es wiederholt zu ausgeprägter Epistaxis. Die Patientin gibt an, unter einem „Leistungsknick“ zu leiden. Sie hat weder Gewicht verloren, noch Fieber oder Nachtschweiß (B-Symptome). Begleiterkrankungen bestehen nicht. Die Familienanamnese bezüglich einer Blutungsneigung ist unauffällig.

Unauffälliger Untersuchungsbefund | Die Patientin ist in einem gutem Allgemeinzustand und schlankem Ernährungszustand (BMI $22 \mathrm{~kg} / \mathrm{m}^{2}$ ). Blutungszeichen fehlen. Die Untersuchung der Thorax- und Bauchorgane ist unauffällig - insbesondere besteht keine palpable Hepatosplenomegalie und kein abdomineller Druckschmerz. Die Frau ist weder ikterisch noch blass oder zyanotisch. Die orientierende neurologische Untersuchung und der Gefäßstatus sind unauffällig. Sie hat keine Ödeme.

\section{Laborparameter}

- Die aktivierte partielle Thromboplastinzeit (aPTT) ist mit 40s leicht erhöht (Norm: 22$34 \mathrm{~s})$.

- Der Quickwert ist mit 78\% (Norm > 70\%) unauffällig.

- Es zeigt sich eine makrozytäre Anämie mit einem Hämoglobinwert von 8,9g/dl (Norm: 12 $16 \mathrm{~g} / \mathrm{dl})$.

- Die Thrombozyten liegen mit $156000 / \mu \mathrm{l}$ im unteren Normbereich (150000-400000/ $\mu \mathrm{l})$.

- Die Leberwerte sind unauffällig.

Die Abklärung der aPTT-Verlängerung ergibt eine deutlich verminderte Faktor-VIII-Aktivität von 25\% (Normbereich 50-150\%). Weitere Ursachen einer aPTT-Verlängerung, insbesondere ein Faktor-XII-Mangel sowie ein Lupusantikoagulans können ausgeschlossen werden. Die sonstigen Faktorenaktivitäten zeigen ein niedrignormales Niveau. Auffällig sind eine deutlich verminderte

- Konzentration [vWF-Antigen: 31\% (Norm: 66$176 \%)]$ und

- Aktivität [Ristocetin-Kofaktor: 34\% (Norm: 61239\%)] des Von-Willebrand-Faktors (VWF).
Die Verschlusszeiten im Platelet-Function-Analyzer (PFA) sind sowohl in der Epinephrin / Kollagenals auch der ADP/Kollagen-Kartusche deutlich verlängert. Die Aggregometrie mit den Agonisten ADP, Arachidonsäure, Kollagen und Ristocetin $(1,5 \mathrm{mg} / \mathrm{ml})$ ist leicht und unspezifisch eingeschränkt. Wir stellen die Verdachtsdiagnose eines erworbenen Von-Willebrand-Syndroms (VWS), wobei die auslösende Grunderkrankung zunächst unklar ist.

\section{Zweite Vorstellung}

Anamnese I Acht Monate später kommt die Patientin erneut zur Diagnostik, weil aufgrund einer Einschränkung des Sehvermögens eine Glaskörperblutung unklarer Genese diagnostiziert wurde. Sie leidet immer noch unter vemehrter Epistaxis.

Labordiagnostik I Jetzt zeigt sich eine stärkere aPTT-Verlängerung auf 47 s. Der Quickwert ist mit $64 \%$ leicht vermindert. Es bestehen eine normozytäre Anämie mit einem Hämoglobinwert von $6,9 \mathrm{~g} / \mathrm{dl}$ und eine Thrombozytopenie von $112000 / \mu l$. Die pathologischen Befunde für die VWF-Parameter lassen sich reproduzieren. Die PFA-Verschlusszeiten sind jetzt sowohl in der Epinephrin/Kollagen- als auch der ADP/Kollagen-Kartusche maximal verlängert (>300s). In der Multimeranalyse sind die normalen Multimere des VWF nicht vorhanden. Die typische Triplet-Struktur der einzelnen Oligomere ist extrem verzerrt. Es ergibt sich der charakteristische Befund eines VWS Typ 2, wobei die Verzerrung der Laufspur in der Elektrophorese typisch für monoklonale IgM-Proteine ist ( Abb. 1).

Ergänzende Untersuchungen I Die zunehmend anämische Patientin wird stationär aufgenommen. Es wird eine monoklonale Gammopathie vom Typ IgM к mit starker IgM-Erhöhung auf $7930 \mathrm{mg} / \mathrm{dl}$ (Norm: 40-230 mg/dl) diagnostiziert. Histologisch zeigt sich eine 50\%ige Knochenmarkinfiltration durch ein lymphoplasmozytoides Lymphom. Die computertomografische Untersuchung des Abdomens zeigt eine mesenteriale Raumforderung $(11 \mathrm{~cm} \times 4 \mathrm{~cm})$. 
Tab. 1 Grunderkrankungen bei erworbenem VWS, nach [2].
Lymphoproliferative
Erkrankungen

Monoklonale Gammopathie (multiples Myelom, M. Waldenström, monoklonale Gammopathie unklarer Signifikanz [MGUS]), Non-Hodgkin-Lymphome, Leukämien (akute und chronische lymphatische Leukämie (ALL,CLL), Haarzellleukämie)

Myeloproliferative Erkrankungen Essenzielle Thrombozythämie, Polyzythämia vera, chronische myeloische Leukämie, Osteomyelofibrose

Tumorerkrankungen

Nephroblastom (Wilms-Tumor), Karzinome und solide Tumoren, peripherer neuroektodermaler Tumor

Immunologische

Erkrankungen

Kardiovaskuläre

Erkrankungen

Sonstiges connective tissue disease, Graft-Versus-Host-Erkrankung

Ventrikelseptumdefekt, Vorhofseptumdefekt, Aortenklappenstenose, Mitralklappenprolaps, Angiodysplasien

Medikamente (Ciprofloxacin, Griseofulvin, Valproinsäure, Hydroxyethylstärke), Infektionen (Epstein-Barr-Virus), andere Systemerkrankungen (Hypothyreose, Diabetes, Urämie, Hämoglobinopathien, Sarkoidose, Teleangiektasien, Colitis ulcerosa, Amyloidose, Ehlers-Danlos-Syndrom)

\section{Diagnose}

Aufgrund der erworbenen Blutungsneigung

- mit Nachweis einer verminderten Konzentration und Aktivität des VWF,

- charakteristischem Befund der Multimeranalyse sowie

- monoklonaler Gammopathie vom Typ IgM к stellen wie die Diagnose eines erworbenen VWS im Rahmen eines M. Waldenströms.

\section{Therapie und Verlauf}

Zu Beginn der Chemotherapie I Die Patienten erhält 5 Zyklen Bendamustin sowie Rituximab (ab dem 2. Zyklus). Darunter nimmt das IgM als Verlaufsparameter ab und die VWF-Parameter normalisieren sich ( $\bullet$ Abb. 2). Parallel steigt die Faktor VIII-Aktivität, bis sich die aPTT normalisiert. Aufgrund der zunächst weiter bestehenden Blutungsneigung erfolgt zur Blutungsprophylaxe eine empirische Therapie mit dem Antifibrinolytikum Tranexamsäure $(2 \times 500 \mathrm{mg} / \mathrm{d})$. Die Dosis wird im Verlauf reduziert und bei Befundverbesserung beendet. Aufgrund einer anämisierenden Epistaxis verbreichen wir der Patientin einmalig Desmopressin. Dadurch kommt es zwar zu einem adäquaten Anstieg der VWF-Parameter, jedoch nicht zu einer Verkürzung der PFA-Verschlusszeiten.

Langsame Normalisierung der Hämostase | Nach zwei Chemotherapie-Zyklen haben sich die VWFParameter normalisiert. Die Blutungsneigung bildet sich vollständig und anhaltend zurück. Im Restaging mittels CT-Thorax/Abdomen zeigt sich nach dem 4. Chemotherapie-Zyklus eine deutliche Befundregredienz. Bemerkenswert ist, dass eine Normalisierung der Verschlusszeiten im PFA erst deutlich später, nämlich erstmals ca. 6 Monate nach Behandlungsbeginn, eintritt.

\section{Diskussion}

Verschiedene Grunderkrankungen | Erstmals wurde 1968 von Simone et al. ein erworbenes VWS bei einem 7-jährigen Patienten mit systemischem Lupus erythematodes beschrieben [1]. Seitdem wurde über viele weitere Fälle im Rahmen verschiedener Grunderkrankungen berichtet.

Seltene Erkrankung I Über Inzidenz und Prävalenz des erworbenen VWS liegen keine exakten Angaben vor. Es handelt sich um eine seltene Erkrankung, welche in der Regel nicht bei Gesunden, sondern als Begleitphänomen im Rahmen einer Grunderkrankung auftritt. Gemäß internationalem Register sind die zugrundeliegenden Erkrankungen überwiegend [2]:

- lymphoproliferative (48\%) und myeloproliferative (15\%) Erkrankungen,

- solide Tumoren (5\%),

- Autoimmunerkrankungen (2\%) und

- kardiovaskuläre Erkrankungen (21\%).

Die wichtigsten Ursachen des erworbenen VWS sind in $>$ Tab. 1 zusammengefasst.

Funktionen des Von-Willebrand-Faktors | Pathophysiologisch ist das erworbene VWS durch eine Dysfunktion des VWF charakterisiert. Dieser vermittelt physiologischerweise bei einer Gefäßläsion die initiale Thrombozytenadhäsion an die subendotheliale Kollagenmatrix. Die Bindung des VWF an Plättchen wird hierbei durch den thrombozytären Rezeptorkomplex Glykoprotein (GP) Ib-V-IX vermittelt. Der VWF kann ferner eine Thrombozytenaggregation unter Beteiligung des thrombozytären Rezeptors GP IIb-IIla vermitteln. Zudem bewahrt er als Trägerprotein den plasmatischen Gerinnungsfaktor VIII vor einer vorzeitigen Proteolyse.

Auslöser | Die Pathogenese des erworbenen VWS ist heterogen. Beschrieben wurden z.B. [3, 4]: 
- gegen den VWF gerichtete Antikörper

- Adsorption des VWF an (maligne) Zellen

- verminderte Synthese des VWF

- vermehrte Spaltung des VWF, etwa bei erhöhten Scherkräften im Rahmen kardialer Vitien Letzterer Mechanismus wird maßgeblich als Ursache für Blutungen aus gastrointestinalen Angiodysplasien bei Aortenklappenstenose verantwortlich gemacht; diese Konstellation wird als Heyde-Syndrom bezeichnet [5].

Bei hämatologischen System-Erkrankungen stellt die Bildung von Antikörpern gegen den VWF den wichtigsten pathogenetischen Faktor bei erworbenem VWS dar.

Diese Antikörper können zum einen spezifisch gegen funktionelle Domänen des VWF gerichtet sein und hierdurch die Funktion des VWF in der Hämostase beeinträchtigen. Zudem können Autoantikörper zur Verkürzung der Halbwertszeit bzw. gesteigerten Clearance des VWF führen [6, 7]. Hiervon zu differenzieren sind unspezifische Antikörper (oft IgM), die an den VWF und andere Plasmaproteine binden. Im berichteten Fall ist denkbar, dass das pathologisch gebildete IgM so zu einem erhöhten Abbau dieser Proteine geführt hat - hierfür spricht die rasche Normalisierung der VWF-Parameter nach Beginn der Chemotherapie.

Blutungen I Wie bei der angeborenen Form kommt es auch beim erworbenen VWS typischerweise zu Schleimhautblutungen, Hautblutungen sowie Blutungen im Rahmen von Eingriffen und Traumata. Muskel- und Gelenkblutungen sind dagegen - im Gegensatz zur Hämophilie - untypisch [4]. Anhand des Blutungsmusters lässt sich das erworbene VWS somit nicht von der angeborenen Form abgrenzen. Anamnestisch tritt beim erworbenen VWS die Blutungsneigung im Laufe des Lebens auf, die frühere Blutungsanamnese des Betroffenen sowie die Familienanamnese bezüglich einer Blutungsneigung sind zumeist unauffällig; in der Regel findet sich eine Assoziation mit einer prädisponierenden Grunderkrankung, die wie im vorliegenden Fall - eventuell erst im Rahmen der Abklärung der Blutungsneigung diagnostiziert wird.

Glaskörperblutungen sehr selten | Glaskörperblutungen [8] als unmittelbare Folge von Gerinnungsdefekten sind untypisch und insbesondere beim VWS eine Rarität. Wir fanden nur zwei Publikationen, in denen das VWS als Ursache von Glaskörperblutungen angesehen wurde: Herrmann et al. berichteten über eine 33-jährige Patientin mit VWS Typ 1 [9]. Shiono et al. (1992) berichteten von einem 13-jährigen Mädchen und einem 19-jährigen Mann, bei denen bei Abklärung einer Glaskörperblutung ein VWS diagnostiziert wurde [10]. Publikationen, in denen ein er-

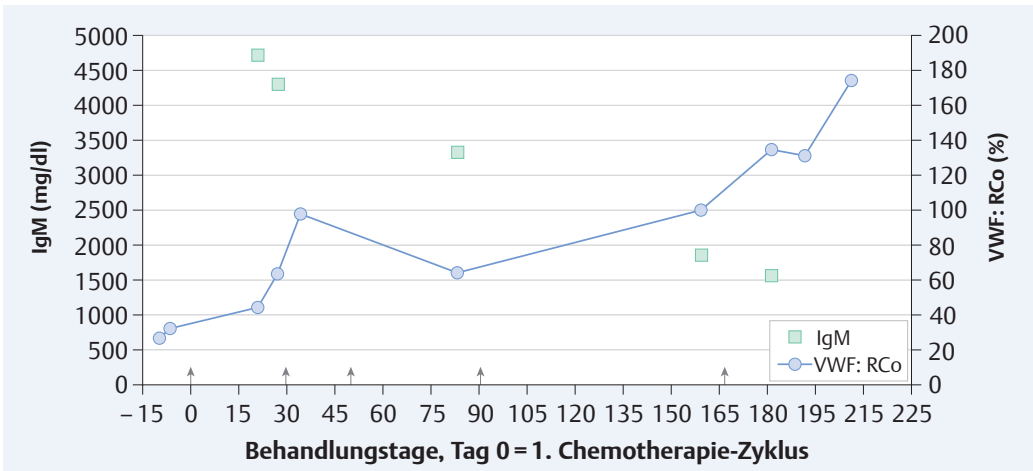

Abb. 2 Verlauf der VWF-Aktivität (Kreis) und von IgM (Quadrat) nach Diagnosestellung mit Angabe der Zeitpunkte der Chemotherapie-Zyklen (Pfeil). 1.Zyklus: Bendamustin $\left(2 \times 70 \mathrm{mg} / \mathrm{m}^{2}\right)$, 2. und 3. Zyklus: Bendamustin $\left(2 \times 85 \mathrm{mg} / \mathrm{m}^{2}\right)$, Rituximab $\left(375 \mathrm{mg} / \mathrm{m}^{2}\right)$, 4. Zyklus: Bendamustin $\left(2 \times 70 \mathrm{mg} / \mathrm{m}^{2}\right)$, Rituximab $\left(375 \mathrm{mg} / \mathrm{m}^{2}\right)$, 5 . Zyklus: Bendamustin $\left(1 \times 70 \mathrm{mg} / \mathrm{m}^{2}\right)$, Rituximab $\left(375 \mathrm{mg} / \mathrm{m}^{2}\right)$.

worbenes VWS zu einer Glaskörperblutung führte, fanden wir nicht.

VWS-Diagnostik | Bei Auftreten einer Blutungsneigung und Vorliegen einer geeigneten Grunderkrankung sollte differenzialdiagnostisch an ein erworbenes VWS gedacht werden. Dieses wird durch die üblicherweise durchgeführte Routinegerinnungsdiagnostik zumeist nicht erkannt.

Zur Diagnose muss eine gezielte VWS-Diagnostik erfolgen, wobei primär Aktivität und Konzentration des VWF zu bestimmen sind.

Hierfür wird zumeist der sogenannte RistocetinKofaktor bestimmt. Eine abnorme Ratio von Aktivität zu Konzentration des VWF (Norm>0.7) kann auf einen funktionellen Defekt des VWF hinweisen. Verlängerte PFA-Verschlusszeiten weisen auf einen Defekt der primären Hämostase hin. Diesbezüglich ist anzumerken, dass die Aussagekraft dieser Methode bei Vorliegen einer Thrombozytopenie limitiert ist, da schon die Thrombozytopenie zu einer PFA-Verlängerung führen kann. Die weiterführende Analytik beinhaltet dann die Durchführung einer Multimeranalyse. Typische Befunde sind ein Verlust der großen VWF-Multimere oder eine verwaschene Triplet-Struktur.

Therapie des erworbenen vws | Bei der Behandlung sind zu unterscheiden [11]:

1. eine (symptomatische) Blutstillungstherapie 2. eine (kausale) Therapie der Grunderkrankung Eine erfolgreiche Therapie der Grunderkrankung führt zu einer Rückbildung des erworbenen VWS mit Normalisierung der VWF-Parameter und Sistieren der Blutungssymptome.

Zusätzlicher Defekt? | Im dargestellten Fall kam es durch Chemotherapie sukzessive zur Normalisierung der VWF-Aktivität ( Abb. 2). Auffällig war, dass die Normalisierung der PFA-Verschlusszeiten erst etwa 6 Monate nach Therapiebeginn eintrat, 


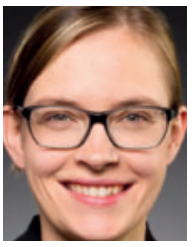

Dr. Stefanie Ackerstaff hat bis Mitte 2013 im LaboMed Gerinnungszentrum in Berlin gearbeitet . Jetzt ist sie wissenschaftliche Mitarbeiterin am Institut für Hämostaseologie, Hämotherapie und Transfusionsmedizin des Universitätsklinikums Düsseldorf.

Stefanie.Ackerstaff@ med.uni-duesseldorf.de

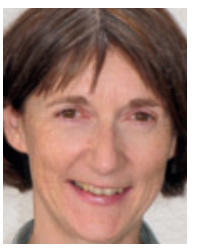

Dr. Rita Dittme

ist Laborfachärztin Hämostaseologin und Vertreterin des Leiters der Abteilung für Hämostaseologie im Zentrallabor der Laborgesellschaft Medilys in Hamburg.

r.dittmer@asklepios.com

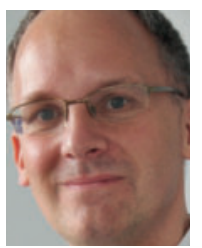

PD Dr. Christoph Sucker ist ärztlicher Leiter und Geschäftsführer des LaboMed Gerinnungszentrums und der Praxis Dr. Sucker in Berlin. sucker@labomed.de

Interessenkonflikt Die Autoren geben an, dass kein Interessenkonflikt besteht.

DOI 10.1055/s-0042-100377 Dtsch Med Wochenschr 2016; 141: 271-274

(c) Georg Thieme Verlag KG . Stuttgart · New York. ISSN 0012-0472 während die Normalisierung der VWF-Aktivität bereits nach ca. 4 Wochen nachweisbar war. Dies kann ggf. dadurch erklärt werden, dass das IgMParaprotein nicht nur die Funktion des VWF, sondern darüber hinaus auch andere Mechanismen der primären Hämostase störte, deren Normalisierung längere Zeit beanspruchte. Das Vorliegen eines zusätzlichen Defektes der primären Hämostase würde auch erklären, dass es nach Desmopressin-Gabe zwar zu einem kurzzeitigen Anstieg der VWF-Parameter kam, die PFA-Verschlusszeiten jedoch maximal verlängert blieben (s.u.).

Symptomatische Blutstillungstherapie I Parallel zur kausalen Therapie muss abhängig von der Schwere der Blutungsneigung eine symptomatische Blutstillungstherapie erfolgen. Hierfür stehen insbesondere zur Verfügung:

- Desmopressin

- VWF-haltige Faktorenkonzentrate

Desmopressin setzt endogenen VWF aus Endothelzellen frei. Beim erworbenen VWS hält der Effekt häufig nur kurz an, bedingt durch eine gesteigerte Clearance des VWF [6, 7].

Im vorliegenden Fall führte die Applikation von Desmopressin (0,3 $\mu \mathrm{g} / \mathrm{kg}$ Körpergewicht als Kurzinfusion) zu einem adäquaten Anstieg der VWFAktivität. Allerdings persistierte eine Störung der primären Hämostase, fassbar durch die persistierend verlängerten Verschlusszeiten im PFA. Dies illustriert, dass hier Desmopressin nicht geeignet war, den erworbenen Gerinnungsdefekt völlig zu kompensieren. Das von uns aufgrund der genannten Blutungssymptome der Patientin zusätzlich eingesetzte Antifibrinolytikum Tranexamsäure ist nicht Standard bei der Blutstillungstherapie beim erworbenen VWS. Der Einsatz ist jedoch als Nebenwirkungsarme und kostengünstige Therapie beim angeborenen VWS üblich und zeigte im präsentierten Fall einen guten Effekt.

Weitere Therapieoptionen | Auch die intravenöse Gabe von hochdosierten Immunglobulinen (IVIG) wurde beim erworbenen VWS im Rahmen lymphoproliferativer Erkrankungen oder monoklonaler Gammopathien erfolgreich eingesetzt, wobei diese Therapie nur bei IgG- und nicht IgM-bildenden monoklonalen Gammopathien therapeutische Erfolge brachte. Der exakte Mechanismus ist unklar - es wird angenommen, dass IVIG mit dem Fc-Rezeptor interagieren und dadurch den Abbau der VWF-Antikörper-Komplexe verlangsamen [3]. Es wurden wenige Fälle publiziert, bei denen eine Plasmapherese zur Therapie des erworbenen VWS eingesetzt wurde. Bei ca. 20\% der Fälle war dies erfolgreich; sehr selten wurde über den Einsatz einer extrakorporalen Immunabsorption berichtet. Immunsuppressive Medikamente und Kortikosteroide waren gemäß internationalem Register bei 19\% der Patienten effektiv, insbeson- dere bei zugrundeliegender Autoimmunerkrankung oder einem mit MGUS assoziierten erworbenen VWS [11].

Konsequenz für Klinik und Praxis

- Das erworbene VWS ist eine seltene, komplexe und heterogene Erkrankung, die mit einer erworbenen Blutungsneigung einhergeht.

- Auch bei atypischen Blutungen ohne alternative Erklärung muss differenzialdiagnostisch an einen erworbenen Gerinnungsdefekt wie ein erworbenes VWS gedacht werden.

- In der Laboranalytik sind eine neu aufgetretene aPTT-Verlängerung bei normalem Quickwert sowie eine Verminderung von Konzentration und Aktivität des VWF wegweisend.

- Eine erfolgreiche Therapie der Grunderkrankung führt in der Regel auch zu einer Remission des erworbenen VWS.

- Bei schwerwiegenden Blutungskomplikationen muss begleitend eine Blutstillungstherapie durchgeführt werden.

\section{Literatur}

1 Simone JV, Cornet JA, Abildgaard CF. Acquired von Willebrand's syndrome in systemic lupus erythematosus. Blood 1968; 31: 806-812

2 Federici AB, Rand JH, Bucciarelli P et al. Acquired von Willebrand syndrome: data from an international registry. Thromb Haemost. 2000; 8: 345-349

3 Veyradier A, Jenkins CS, Fressinaud E et al. Acquired von Willebrand syndrome: from pathophysiology to management. Thromb Haemost. 2000; 84: 175-182

4 Sucker C, Stockschläder M, Zotz RB et al. Das erworbene von-Willebrand-Syndrom. Dtsch Med Wochenschr. 2004; 129: 1581-1585

5 Sucker C. The Heyde syndrome: proposal for a unifying concept explaining the association of aortic valve stenosis, gastrointestinal angiodysplasia and bleeding. Int J Cardiol 2007; 115: 77-78

6 Kumar S, Pruthi RK, Nichols WL. Acquired von Willebrand disease. Mayo Clin Proc 2002; 77: 181-187

7 Sucker C, Michiels JJ, Zotz RB. Causes, etiology and diagnosis of acquired von Willebrand disease: a prospective diagnostic workup to establish the most effective therapeutic strategies. Acta Haematol 2009; 121: 177-182

8 Grehn F. Augenheilkunde. 30. Aufl. Heidelberg: Springer; 2008: 259

9 Herrmann WA, Lohmann CP, Demmler-Hackenberg $\mathrm{M}$ et al. Von Willebrand's disease type I as a cause for subvitreal, retinal and subretinal haemorrhages. Graefes Arch Clin Exp Ophthalmol 2005; 243: 383-385

10 Shiono T, Abe S, Watabe T et al. Vitreous, retinal and subretinal hemorrhages associated with von Willebrand's syndrome. Graefes Arch Clin Exp Ophthalmol 1992; 230: 496-497

11 Franchini M, Lippi G. Acquired von Willebrand syndrome: an update. Am J Hematol 2007; 82 368-375 